\title{
Personalised/precision medicine
}

\author{
Shaun R. McCann* \\ Professor Emeritus of Haematology and Academic Medicine, St James' Hospital and Trinity College Dublin, Ireland
}

The genetic revolution began in 1953 with the discovery by Watson and Crick of the DNA double helix [1]. Following closely on that observation Sickle Cell Disease was the first 'molecular' disease to be described, the substitution of Valine for Glutamic acid on the $\beta$ chain of the globin protein with devastating consequences for the affected individual. Since then haematology has been in the forefront of 'molecular' diseases presumably, in part, due to the ease of obtaining samples in benign and malignant diseases. There is no doubt that the polymerase chain reaction greatly facilitated the development of the concept of 'minimal residual disease' or 'measurable residual disease' initially in Follicular Lymphoma then Chronic Myeloid Leukaemia (CML) and now Acute Lymphoblastic Leukemia The development of inexpensive and widely available tools for gene sequencing has undoubtedly furthered the revolution. A note of caution however should be injected. As Sir Gordon Duff said following the unraveling of the human genome: 'we have the alphabet, now we need the language' $[2]$.

From the patient's perspective the 'genetic revolution' resulted in the development of specific tyrosine kinase inhibitors (TKIs) for the treatment of patients with CML. CML is characterized by the reciprocal translocation of $\mathrm{ABL}$ on chromosome 9 to become juxaposed to $\mathrm{BCR}$ on chromosome 22 thereby creating a chimeric gene with deregulated ABL kinase activity, which was subsequently shown to control the malignant phenotype. As this reciprocal translocation is present in all patients with CML (irrespective of the transcript size) this disease is ideally suited to 'targeted therapy'. This development has revolutionized the treatment of CML and many patients are alive and well almost 20 years since the diagnosis of what was previously a universally fatal illness. The question now is: 'can patients safely discontinue TKIs and remain in 'remission from their CML? This success story has led to the quest to understand the molecular nature of disease and hopefully eventually a cure. The second question is: 'Is CML a paradigm for cancer or just an unconventional disease?'.

As CML is a relatively uncommon disease compared to breast or colon cancer the response to TKIs has heralded the search for molecular lesions in many diseases and resulted in the concept of personalised/ precision medicine. What is meant by personalised/precision medicine is interventions to prevent, diagnose or treat a disease (e.g. cancer) based on a molecular and/or mechanistic understanding of the pathogenesis and/or pathology of the disease.

Therefore where the individual characteristics of a patient are sufficiently distinct interventions can be concentrated on those who will benefit, sparing expense and side effects to those who will not. Many people believe that precision medicine will lead to a fundamental understanding of the complex interplay between genetics, epigenetics, nutrition, environment and clinical presentation thus facilitating the development of effective prevention and treatment.
The development of combination chemotherapy in the 1950s and the rapid growth of new technologies to evaluate molecular, genetic and epigenetic changes in malignant cells have inexorably led to the idea of personalised medicine. The 'one size fits all' philosophy which underpins combination chemotherapy may seem a little naive now and it is clear that some patients will not respond to treatment while others with ostensibly the same disease, will have a complete remission. A similar attitude can be taken to randomise clinical trials, for so long the 'gold standard' for evaluating the effects of drugs. Sub-group analyses of individuals whose disease has a similar genetic profile may show an excellent response to treatment which may be lost if the group is evaluated as a whole. However, if these analyses are retrospective they should be validated by prospective studies.

When associated with pharmacogenetics personalised/precision medicine can also apply to non-malignant diseases. The term personalised medicine may be misleading as it does not mean that treatment will be 'personalised' for each individual but where groups of patients, usually with malignant disease, share genetic/molecular/ epigenetic abnormalities then treatment may be tailored for that group. In the future malignant tumors may no longer be classified based on the organ of origin (lung cancer, breast cancer, prostate cancer, etc.) but rather on their common genetic characterization (e.g. BRCA1/2 mutation which occurs in a significant fraction of patients suffering from breast, pancreas, colon, prostate cancer, etc.). As Professor Anton Hagenbeek says:' one of the utmost advantages of personalised medicine is to prevent harm to the majority of patients' [3]. A recent publication in the New England Journal of Medicine provides a flavour of what future clinical trials might look like. In this trial patients with breast cancer were stratified to receive chemotherapy or not on the basis of 'gene profiling' [4]. If the data are confirmed in other trials and with longer follow up then Hagenbeek's words may be prophetic.

In 2010 Margaret Hamburg and Francis Collins (Commissioner of the FDA and Director of the NIH respectively) wrote a 'perspective' about personalised medicine in the New England Journal of Medicine [5] in which they said that at that time there was insufficient evidence to interest the pharmaceutical industry to invest in this type of research but that the NIH would promote it through programs such as the Therapeutics for Rare and Neglected Diseases (TRND). They hoped that programs like TRND would lead to discovery of molecular subtypes of common diseases which might support new therapies. They stressed the importance of biobanks which could provide tissues for genomic evaluation. The authors issued words of caution, however. The success

Correspondence to: Shaun R. McCann, Professor Emeritus of Haematology and Academic Medicine, St James' Hospital and Trinity College Dublin, Ireland, Tel: +353 1896 3236; E-mail: shaunrmccann@gmail.com

Received: July 29, 2016; Accepted: August 24, 2016; Published: August 27, 2016 
of personalised medicine would depend on accurate diagnostic tests and they pointed out that many of these tests have not been verified by independent investigators. Non-validated tests may miss the genetic abnormality which could advocate early intervention or could over diagnose the risk of malignancy. However in the intervening period many Pharma companies have indeed pursued the development of socalled 'targeted therapy'.

There is a large amount of interest in the concept of personalised medicine amongst the public, media and, to a lesser extent, the medical profession. However there is a European Personalised Medicine Association, EPEMED and a Personalised Medicine Coalition whose aims are to share vital insights into personalised medicine and to provide a platform for the harmonization of developments and implementation of personalised medicine. The $3^{\text {rd }}$ Astellos Innovation Debate (Astellas Pharma is a Japanese Phermaceutical company founded in 2005) 'What the DNA and Data revolution means for our health' was held in London, UK in January 2015. The debate, held in the Royal Institute London, UK, was moderated by journalist Jonathan Dimbleby. George Freeman MP, Life Sciences Minister in the UK government said that the National Health Service (NHS) in the UK would collect molecular/genetic data on 100,000 patients and match these with the patients' medical charts. The information will then be shared with the Pharma industry in an anonymised fashion. Whether this type of approach will yield useful results or not remains to be seen. Many people have grave doubts about the wisdom of agencies such as the NHS collecting large amounts of data on individuals as people fear that the information might be used for nefarious purposes or might fall into the wrong hands. The public also fears that data can never be truly anonymised. If the Pentagon can be 'hacked' then surely no data base is truly secure! Reporting in the International New York Times on August $17^{\text {th }} 2016$ Edward Wong says that the first quantum communications satellite launched by China may lead to a completely secure method of transmitting information. Collecting genomic data on tumours is generally accepted but collecting data on the whole genome of patients is hotly disputed.

Many interesting issues were raised in the debate in London but two, in particular, stand out. Firstly the attitude to privacy seems to be a generational one. Young people do not have the same regard for privacy as the over $60 \mathrm{~s}$ as witnessed by the posting of private information in social media by younger people. If genomic analyses are to become the 'norm' then it will require a complete overhaul of medical education and the education of thousands of genetic counsellors. Secondly as Professor Tarassenko (a debate pannelist) pointed out the ability of a sophisticated and presumably highly-educated audience to understand the mathematics of 'probability' and concurrent probabilities is limited to about $5 \%$. How then can we expect the public to understand these statistical concepts? [6] Personalised medicine would also change the 'doctor-patient relationship' which we have believed in for over two thousand years. In response to a very interesting question posed by Jonathan Dimbleby the audience was overwhelmly opposed to the sequencing of an individual's genome at birth but a majority believed, at the same time that this would happen.

Although the public and the media have embraced the idea of 'personalised medicine' the medical profession does not seem to be as enthusiastic is this because doctors are afraid that patients will know more about their disease and genome than the doctor? Will the relationship between doctor and patient be altered by this type of information being made available to the patient? To a certain extent this may already be happening with telephone and Skype consultations. The answers to these questions may become clear in the next decade if and when personalised medicine becomes a reality for large numbers of patients.

Medical practise has made many promises in the past, some unfulfilled and some altering the outcome for many diseases. Combination Chemotherapy has not been successful in the majority of solid tumours and gene therapy has yet to be widely applied. Monoclonal antibodies have been startlingly successful in the treatment of nonHodgkin lymphoma but their development took over 30 years. While the molecular and personalised approach to medicine may herald cure for many patients we should be cognisant of the words of Niels Bohr, Nobel Prize Laureate for Physics in 1922 who said: 'Prediction is difficult especially if it is about the future'.

\section{References}

1. Watson JD, Crick FH (1953) Molecular structure of nucleic acids; a structure for deoxyribose nucleic acid. Nature 171: 737-738. [Crossref]

2. Duff G (2014) Validity and sensitivity of the phonics screening check: implications for practice. Personal communication 38: 109-123.

3. Hagenbeek A (2015) Personal communication.

4. Cardoso F, van't Veer LJ, Bogaerts J, Slaets L, Viale G, et al. (2016) 70-Gene Signature as an Aid to Treatment Decisions in Early-Stage Breast Cancer. N Engl J Med 375: 717-729. [Crossref]

5. Hamburg MA, Collins FS (2010) The path to personalized medicine. $N$ Engl J Med 363: 301-304. [Crossref]

6. The Astellas Innovation Debate (2015) What the DNA and Data Revolution mean to our Health.

Copyright: $\odot 2016 \mathrm{McCann}$ SR. This is an open-access article distributed under the terms of the Creative Commons Attribution License, which permits unrestricted use, distribution, and reproduction in any medium, provided the original author and source are credited. 